\title{
Incentivizing High Quality Crowdwork
}

\author{
Chien-Ju Ho* \\ University of California, Los Angeles \\ cjho@ucla.edu
}

\author{
Siddharth Suri \\ Microsoft Research \\ suri@microsoft.com
}

\author{
Aleksandrs Slivkins \\ Microsoft Research \\ slivkins@microsoft.com \\ Jennifer Wortman Vaughan
Microsoft Research
jenn@microsoft.com
}

\begin{abstract}
We study the causal effects of financial incentives on the quality of crowdwork. We focus on performance-based payments (PBPs), bonus payments awarded to workers for producing high quality work. We design and run randomized behavioral experiments on the popular crowdsourcing platform Amazon Mechanical Turk with the goal of understanding when, where, and why PBPs help, identifying properties of the payment, payment structure, and the task itself that make them most effective. We provide examples of tasks for which PBPs do improve quality. For such tasks, the effectiveness of PBPs is not too sensitive to the threshold for quality required to receive the bonus, while the magnitude of the bonus must be large enough to make the reward salient. We also present examples of tasks for which PBPs do not improve quality. Our results suggest that for PBPs to improve quality, the task must be effort-responsive: the task must allow workers to produce higher quality work by exerting more effort. We also give a simple method to determine if a task is effort-responsive a priori. Furthermore, our experiments suggest that all payments on Mechanical Turk are, to some degree, implicitly performance-based in that workers believe their work may be rejected if their performance is sufficiently poor. Finally, we propose a new model of worker behavior that extends the standard principal-agent model from economics to include a worker's subjective beliefs about his likelihood of being paid, and show that the predictions of this model are in line with our experimental findings. This model may be useful as a foundation for theoretical studies of incentives in crowdsourcing markets.
\end{abstract}

\section{Categories and Subject Descriptors}

\section{J.4 [Social and Behavioral Sciences]: Economics}

\footnotetext{
${ }^{*}$ Much of this research was conducted while Ho was visiting Microsoft Research.
}

Copyright is held by the International World Wide Web Conference Committee (IW3C2). IW3C2 reserves the right to provide a hyperlink to the author's site if the Material is used in electronic media.

$W W W$ 2015, May 18-22, 2015, Florence, Italy.

ACM 978-1-4503-3469-3/15/05.

http://dx.doi.org/10.1145/2736277.2741102

\section{General Terms}

Economics, Experimentation

\section{Keywords}

Crowdsourcing; Performance-Based Payments; Incentives

\section{INTRODUCTION}

Crowdsourcing markets are platforms on which workers around the world perform tasks for pay. In a crowdsourcing market like Amazon Mechanical Turk, requesters post tasks along with the amount of money that they are willing to pay workers for their task's completion. Workers can then browse the available tasks and choose tasks to work on.

Crowdsourcing markets are used to conduct user studies [22], run behavioral experiments [18, 28], collect data [17] 35, test or even build business applications [1, 31, and more. While these markets are effective at recruiting diverse labor pools, the quality of work produced varies widely across tasks and workers. The prevalence of low quality crowdwork has inspired a growing literature on techniques to boost accuracy, for example, by using redundant assignments for labeling tasks [15, 19, 21, 26, 34, smartly assigning tasks to workers 14, 15, introducing social incentives 30, 33, or altering financial incentives $4,10,12,29,30,33,36,37$. These solutions have had mixed success, and how to improve the quality of work in general is still not well understood.

In this paper, we study the use of financial incentives to encourage high quality crowdwork on Amazon Mechanical Turk. In particular, we focus on the use of performancebased payments (PBPs), bonus payments awarded to workers for producing high quality work. Previous empirical studies of performance-based payments in crowdsourcing markets have produced mixed and somewhat contradictory recommendations. Harris 12 and Yin et al. 37 suggested that PBPs can improve work quality, while Shaw et al. 33. found no improvement and Yin et al. 36 found no difference in quality when varying bonus size.

Our results explain these disparities in prior work. Furthermore, we show how to generalize previous findings beyond the particular tasks that were studied. We design and run experiments with the goal of understanding not just whether PBPs improve work quality for a specific task or bonus size, but when, why, and where they improve work quality. We identify properties of the payment, payment structure, and the task itself that make PBPs effective. 
In our experiments, we first identified a task (proofreading an article) for which PBPs improve workers' performance. We tested the robustness of this finding by varying the payment structure and amount. We found that using PBPs with a wide range of quality thresholds improved work quality provided the bonus awarded for exceeding the threshold was sufficiently high. We also found that even when standard, unconditional payments are used and no explicit acceptance criteria is specified, workers may behave as if the payments are implicitly performance-based since they believe their work may be rejected if its quality is sufficiently low.

We examined potential reasons why PBPs improve quality. We found that simply increasing the amount of the base payment without offering any bonus significantly improved quality, contradicting several previous studies [2, 4, 10, 29, 30. However, PBPs led to improved quality and lower cost compared to a guaranteed payment of the same amount. We also found that whether the opportunity to receive a bonus or higher base payment is revealed before or after the task is accepted does not make a difference in the quality of crowdwork, ruling out the possibility that the increased quality we observed was due (at least in part) to the reciprocity caused by workers' joy at receiving an unexpected bonus, as discussed by Gilchrist et al. 10.

Finally, we investigated which properties of a particular task allow PBPs to have an effect. We examined the conjecture that PBPs are more likely to improve quality on effort-responsive tasks, tasks for which workers can produce higher quality work by exerting additional effort. We ran experiments on four different tasks. By taking the amount of time workers spent on the task as the proxy measure for their effort, we found that additional effort was correlated with improved quality for the tasks for which PBPs helped, but not for the tasks for which PBPs did not help. This observation yields a simple method for requesters to determine whether or not a given task is likely to benefit from PBPs.

Based on our experimental results, we propose a simple theoretical model of worker behavior. The model is a variant of the standard principal-agent model from economics that additionally incorporates workers' subjective beliefs about the quality of work required to be paid. We show that this model can be used to explain our key empirical observations which cannot be explained using the principal-agent model alone. This model may be useful as a more realistic foundation for future theoretical work on crowdsourcing markets.

Related Work. Performance-based payments have been studied extensively outside of crowdsourcing markets. Lazear 24] conducted a highly influential analysis of observational data from an autoglass company that switched from paying workers a fixed hourly rate to paying workers based on the number of units installed, and showed that workers' performance significantly improved when payments were contingent on work done. As another example, Gneezy and Rustichini 11] gave college students fifty questions from IQ tests to answer and found that their performance increased when they received a bonus for answering questions correctly as long as the bonus was sufficiently high. On the other hand, when the bonus payment was very small, quality decreased compared with not offering a bonus at all. The psychology literature suggests that this is perhaps due to a decrease in workers' intrinsic motivation (enjoyment, responsibility, pride) for performing well, though this theory is not universally accepted 6, 8, 9.

Camerer and Hogarth 5] performed a meta-analysis of 74 papers examining the effect of using payments contingent on performance in lab experiments. They showed that using payments contingent on performance improved the average performance of subjects for tasks in which increased effort leads to improved performance, such as memory or recall tasks, clerical tasks such as coding words or building things, and problem-solving tasks. Similar meta-analyses were performed by Jenkins Jr. et al. [20], Bonner et al. [3], and Hertwig and Ortmann 13 . We build on and extend this work by showing that PBPs have a causal impact on work quality in a field setting, specifically, a crowdsourcing environment in which eliciting high quality work is a main concern for requesters. Moreover, we give evidence to support the conjecture of Camerer and Hogarth [5] that PBPs work in tasks that are effort-responsive.

The results of studies on performance-based payments in crowdsourcing markets have been mixed and sometimes discouraging. On the positive side, in an early workshop paper, Harris [12] showed that when asking workers to evaluate the relevance of resumes, PBPs increased both performance and the time workers spent on the task. In very recent work, Yin et al. 37. studied a setting in which workers switched back and forth between two types of tasks, and showed that PBPs improved performance, especially when used immediately after a task switch.

On the negative side, Shaw et al. 33. compared fourteen different incentive schemes, including four using PBPs ${ }^{1}$ and saw little variation in their effects on quality of work. However, the bonuses offered were extremely small, only $10 \%$ (\$0.03) on base payments of $\$ 0.30$. One might hypothesize that the lack of effect stems from decreased intrinsic motivation as in Gneezy and Rustichini [1], or that obtaining the small bonus is simply not worth the costly additional effort that would be required. More recently, Yin et al. 36. studied the effect of varying the bonus size of PBPs and found that the size of the bonus did not impact the quality of work. In their case, all bonuses offered were large compared with the base payment $(\$ 0.04, \$ 0.08, \$ 0.16$, or $\$ 0.32$ on a base payment of $\$ 0.01$ per task), so it is possible that even their smallest bonus was large enough to elicit the workers' maximum effort; since Yin et al. did not include a treatment without PBPs, there is no way to know whether PBPs boosted quality compared with offering base payments alone. In Section 4 we give a unifying explanation for these results. We show small bonuses in a PBP result in little to no effect on work quality, and that it can be hard to detect the effect between two large PBP bonuses. However, the overall trend is that using PBPs with sufficiently high bonuses yields better quality work.

Several additional studies have examined financial incentives in crowdsourcing markets. Horton and Chilton 17 empirically estimated workers' reservation wages and found

\footnotetext{
${ }^{1}$ The differences in these four treatments were whether payments were described in terms of rewards for high quality or punishments for low quality, and whether quality was measured objectively or in terms of agreement with other workers' responses. Of these, the only treatment that resulted in performance statistically significantly different than the control was punishing workers when their responses did not agree with others.
} 
that many workers aim to hit payment targets (such as multiples of $\$ 0.05$ ). Mason and Watts 29 found that paying workers more increased the number of tasks workers chose to complete, but did not increase performance on each task. That increased pay did not increase performance was observed by other authors as well [4, 10, 25, 30. In this paper, we found that paying workers more can actually increase their performance for some types of tasks. This disparity can be explained by considering workers' subjective beliefs on how much work they must do to get their work accepted. In prior work, workers either already performed well even with low pay since the tasks were easy or were given additional instructions which could have primed their subjective beliefs. In our experiments, workers are uncertain about how much work they should do to get paid. Therefore, they are willing to produce higher quality work to increase their chance of having their work accepted when the payments are higher.

Gilchrist et al. [10] showed that the manner in which the payment is presented can influence quality. In particular, they found that on oDesk, a crowdsourcing market for larger tasks, initially telling workers they would receive $\$ 3$ per hour and increasing this payment to $\$ 4$ after the job was accepted led to higher performance than paying either $\$ 3$ or $\$ 4$ per hour without the surprise. We show in Section 5 that this result did not translate to the Mechanical Turk setting.

Ho et al. [16] studied the algorithmic problem of adaptively optimizing PBPs in crowdsourcing markets, modeling the problem as a dynamic variant of the standard principalagent model from contract theory. Their model assumed that each worker chooses how much effort to exert in order to maximize his expected utility, which is simply his expected payment minus the cost of his effort. In Section 7 . we propose a variant of this worker model that is in line with our experimental observations. The results of Ho et al. 16] still apply when our worker model is used in place of theirs.

\section{PRELIMINARIES}

Before describing our experiments, we briefly describe the setting in which they were conducted. All of our experiments were run on Amazon Mechanical Turk (henceforth MTurk), one of the most popular crowdsourcing platforms. On MTurk, requesters post tasks (HITs) for workers to complete. When a worker browses a task, he sees a description of the work to be done along with the amount of money that the requester has offered as a base payment. A worker can then choose whether to accept the task. After the worker completes the chosen task, the requester may evaluate the worker's submission and choose to either approve or reject the work. Each worker has an approval rating which is simply the fraction of HITs he has submitted that have been accepted. If the work is rejected, the worker is not paid and his approval rating, which serves as a de facto reputation score, suffers. If the work is accepted, the worker receives the base payment for the task and his approval rating increases. Requesters also decide at this time whether or not to award a bonus payment on top of the base, and how much of a bonus to award. The possibility of such a bonus may or may not be included in the task description.

HITs can also have qualifications associated with them. Only those workers who have the appropriate qualifications

\footnotetext{
${ }^{2}$ https://mturk.com
}

can do a HIT. These qualifications are specified by the requester. For example, we used a geographic qualification to restrict our tasks to workers located in the United States, and used qualifications to disallow workers from completing the same type of task more than once. We also used qualifications for random assignment in one of our experiments; how and why we did this is described in Section 5

Our experiments focus on the use of threshold-based PBPs. A threshold-based PBP is specified by a base payment, a bonus payment, and a threshold. The base payment specifies the amount of money a worker receives from completing the task; this is fixed at $\$ 0.50$ USD in all of our experiments ${ }^{3}$ The bonus payment specifies the amount the worker can potentially receive as a bonus. The threshold determines what the worker must do in order to obtain the bonus.

All of the experiments in this paper were approved by the Microsoft Research IRB.

\section{DOES PBP WORK?}

Our first experiment was designed with two goals in mind. The first was to verify that PBPs can lead to higher quality crowdwork and identify a task for which this happens. The second was to determine if there exists what we call an implicit PBP effect: even if the requester offers a guaranteed payment, MTurk workers have subjective beliefs on the quality of work they must produce in order to receive this payment, and therefore behave as if the payments were (implicitly) performance-based. We measure these subjective beliefs by the difference in the quality of crowdwork when base payments are explicitly guaranteed, effectively resetting the workers' subjective beliefs, compared to when base payments are not guaranteed.

\subsection{Experiment Design}

In this experiment, workers were asked to proofread an article of 500 to 700 words and correct spelling errors. For each article, we randomly inserted 20 typos from a list of common spelling errors. Workers were asked to input the line number of each typo, the misspelled word, and the correct spelling of the word.

This task has two key properties. First, we would expect that workers could produce better work by exerting more effort - the more carefully a worker reads or the more passes a worker takes over the text, the more typos he will find - and that this would open up the possibility of PBPs improving quality. (We study this conjecture in more detail in Section 6.) Second, since we injected the typos into the text, the quality of each worker's output could be measured objectively, though this was not known to the workers.

Before accepting the HIT, each worker saw a preview consisting of the instructions, an example article with typos, and the base payment of $\$ 0.50$ USD. The preview was the same for all workers. After workers accepted the HIT, they were randomly assigned to different treatments and then shown treatment-specific instructions, when applicable. Our experiment had a $2 \times 3$ design, with 2 treatments governing the base payment and 3 treatments governing the bonus payment (if any). We discuss the bonus treatments first:

\footnotetext{
${ }^{3}$ The base payment of $\$ 0.50$ was chosen so that workers could obtain a $\$ 6$ hourly rate from the base payment alone with a reasonable amount of effort in each of our tasks.
} 
- No Bonus: This is the control group. It had no bonus and no mention of a bonus.

- Bonus for All: All workers earned a $\$ 1$ bonus after submitting the HIT.

- PBP: Workers earned a $\$ 1$ bonus if they found $75 \%$ of the typos found by the other workers.

The purpose of the control group was to allow us to measure the baseline number of typos workers found. The purpose of the Bonus for All treatment was to test if simply paying more resulted in higher quality work. The purpose of the PBP bonus treatment was to test if specifically incentivizing for quality improved the number of typos found.

As mentioned, we are also interested in whether workers have subjective assumptions on how much effort they must exert to get their work accepted. Workers may be afraid that if they do not find a sufficient number of typos their work will be rejected, resulting in no pay and a negatively affected MTurk reputation. To estimate this, we designed a treatment in which workers were explicitly guaranteed acceptance provided that they completed a very small amount of work. We had two treatments for the base payment:

- Non-Guaranteed: There were no extra instructions. This is the control and emulates most MTurk tasks.

- Guaranteed: Workers were told they would get paid if they found at least one typo.

The first typo appeared before line 3 in each article. Thus a worker would only have to do a trivial amount of work to ensure they got paid in the guaranteed base treatment.

\subsection{Results}

The HIT was completed by 1,000 unique workers, who were each assigned uniformly to one of the six treatments. We conducted a chi-squared test to check for significant differences in the number of participants who finished the six treatments and found none $(p=0.38)$. The primary dependent variable (or outcome measure) we were interested in is the number of true typos found. In the analysis we made six comparisons that we spell out below. We performed this analysis using an ANOVA with one-sided, planned comparisons [32] and report p-values that have been corrected for these multiple (six) comparisons. The results of this experiment are shown in Figure 1 and described below.

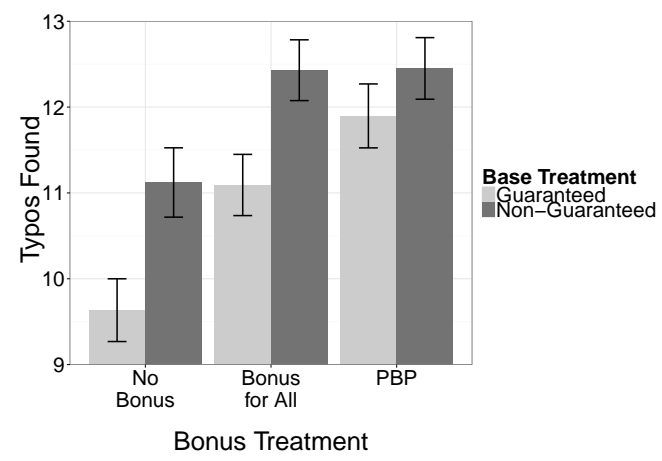

Figure 1: The effect of different payment schemes on work quality in the proofreading task. Error bars indicate the mean \pm one standard error.
PBPs improve quality. To determine whether PBPs increase quality for this task, we focus on the non-guaranteed base treatments since almost all HITs on MTurk do not explicitly guarantee any kind of acceptance criteria. Workers in the PBP bonus treatment found on average 1.3 more typos than workers in the No Bonus treatment $(p=0.042)$, showing that PBPs did improve quality for this task.

All payment schemes may be implicitly performancebased. In the No Bonus treatment, the guaranteed base resulted in 1.5 fewer typos found on average compared with the non-guaranteed base $(p=0.015)$. Similarly, in the Bonus for All treatment, the guaranteed base resulted in 1.3 fewer typos found on average $(p=0.024)$. While there may be other explanations, this suggests that workers do have subjective beliefs on the amount of work that needs to be done for their work to be accepted, lending support to our conjecture that payments on MTurk are already implicitly performance-based. We discuss this further in Section 7

In the PBP bonus treatment, we did not see a significantly different effect between the guaranteed base and nonguaranteed base treatments. We offer two related explanations of this finding. First, the only way to grant a bonus using the MTurk API is to first accept the work. This means that in the PBP bonus treatment, workers would likely believe that finding $75 \%$ of typos would almost certainly result in their work being accepted, already altering their subjective beliefs. Second, the treatment might have made this $75 \%$ threshold more salient to the workers. This gave a clear goal for the workers to strive for.

Simply paying more improves quality. Focusing again on the non-guaranteed base treatment, workers in the Bonus for All treatment found on average 1.3 more typos than workers in the No Bonus treatment $(p=0.036)$. Thus offering an unconditional bonus - which is essentially just paying more-increased quality.

This finding is perhaps surprising since it appears to contradict the results of prior work [4, 29, 30. We give two potential explanations. First, since the announcement of the bonus came after workers accepted the HIT, the workers may be exhibiting reciprocity by doing higher quality work [10], rewarding the requester for this pleasant surprise. We further test and refute this hypothesis in Section 5. Second, this could be explained by the implicit PBP effect described above. That is, workers might have subjective beliefs about the number of typos they must find to get paid. If we increase the bonus payment, workers might be willing to put in more effort to increase their probability of earning this higher amount.

This observation is not inconsistent with previous work. In most prior work, either easy tasks were chosen which might cause workers to perform well even for low pay 4, 30. or additional instructions or tutorials were provided which may have primed workers' subjective beliefs 29.

PBPs can save money compared with high unconditional payments. In the non-guaranteed base treatment, the difference in the number of typos found in the PBP and Bonus for All treatments is not significant. Both resulted in higher quality work than the control. However, we spent much less money on the PBP treatment. We paid each worker $\$ 1.50$ in the Bonus for All treatment, while we paid each worker only $\$ 0.97$ on average in the PBP treatment 
with non-guaranteed base and $\$ 0.96$ on average in the PBP treatment with guaranteed base. Therefore, it may still be advantageous for requesters to offer PBPs even if they could achieve the same quality work with unconditional payments.

\section{WHEN DOES PBP WORK?}

Having established that PBPs can improve quality for the proofreading task, we investigated the effect of varying two parameters of the payment scheme, the bonus threshold and the bonus amount, to better understand when PBPs help.

\subsection{Bonus Thresholds: Experiment Design}

We first tested the effect of varying the threshold of quality that must be met in order for workers to receive the bonus. We used the same proofreading task described in Section 3.1. with the same base payment of $\$ 0.50$ and bonus of $\$ 1$. Workers were randomly assigned to treatments in which they were told they could earn the bonus if they found at least 5 typos or at least $25 \%, 75 \%$, or $100 \%$ of the typos found by the other workers. In the control, workers did not receive any bonus or see any mention of a bonus.

\subsection{Bonus Thresholds: Results}

The results from 585 unique workers are presented in Figure2. As before, we ran an ANOVA with one-sided, planned comparisons 32 and report p-values that have been corrected for the multiple (five) comparisons we describe below.

PBPs improve quality for a wide range of bonus thresholds. Improvements in quality over the control can be observed in both the $25 \%$ and $75 \%$ treatments. The $25 \%$ treatment resulted in workers finding, on average, 1.1 more typos than the control $(p=0.082)$. Similarly, the $75 \%$ treatment resulted in workers finding, on average 1.2 more typos, than the control $(p=0.049)$. We conjecture that setting a threshold anywhere between $25 \%$ and $75 \%$ would yield similar results, so the improvements from PBPs are not overly sensitive to the threshold. However, the $100 \%$ typo condition was neither significantly different than the $75 \%$ treatment or control, so our results suggest that if the bonus threshold is set too high, then workers' average performance slightly decreases. This could be due to some workers giving up because they do not believe the bonus is attainable.

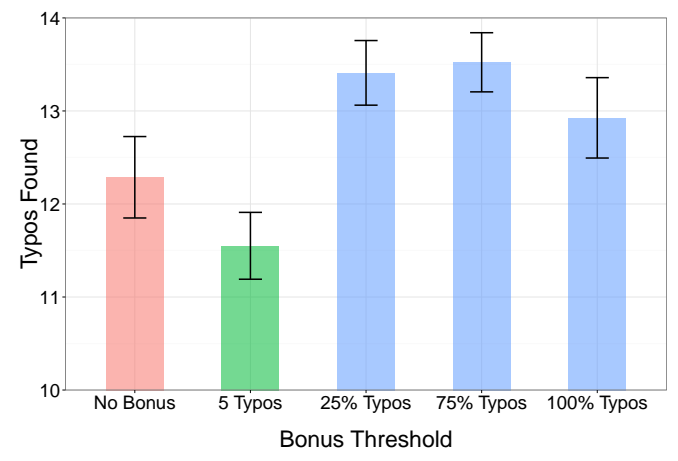

Figure 2: The effect of the bonus threshold on work quality in the proofreading task. Error bars indicate the mean \pm one standard error.

Subjective beliefs on the quality thresholds can improve work. Since each article contains 20 typos in total,
$25 \%$ of the typos found by other workers is at most 5 typos and will be exactly 5 typos if the number of workers is sufficiently large. In this sense, the two thresholds are roughly equivalent. However, workers in the $25 \%$ treatment performed much better than workers in the 5 typos treatment. Workers in the $25 \%$ treatment found, on average, 1.9 more typos than those in the 5 typo treatment $(p<.001)$. An analysis of the line numbers in which typos were found showed that workers in the 5 typo treatment did not stop reading before workers in the $25 \%$ treatment; however, there may be a variety of other explanations for this. For example, it is possible that workers in the $25 \%$ group had different subjective beliefs and thought that they would need to find more than 5 typos to receive the bonus. After all, workers did not know the total number of typos in the article.

\subsection{Bonus Amounts: Experiment Design}

We next examined the effect of varying the bonus amount. We used the same proofreading task with a base payment of $\$ 0.50$. Workers were assigned to treatments in which they could earn either $\$ 0.05, \$ 0.50$, or $\$ 1$ if they found $75 \%$ of the typos found by other workers. Once again, workers in the control did not receive or see any mention of a bonus.

As an implementation detail, we note that these experiments were run simultaneously with those described in Section 4.1. allowing us to share two treatments (the control and the $\$ 1$ for $75 \%$ ) and run only seven treatments in total instead of nine. We excluded workers who had already participated in the experiment from Section 3. We collected results from 815 unique workers assigned uniformly to the seven treatments. A chi-squared test showed no significant differences between the number of workers who completed the seven treatments $(p=0.23)$

\subsection{Bonus Amounts: Results}

We collected data from 451 unique workers. Figure 3 shows the overall trend: PBPs lead to higher quality work only when the bonus is sufficiently large, but increasing the bonus amount has diminishing returns. Indeed, regressing the number of typos found on the bonus amount shows that an extra $\$ 1$ of bonus results in finding 1.4 more typos $(p=0.002)$ on average.

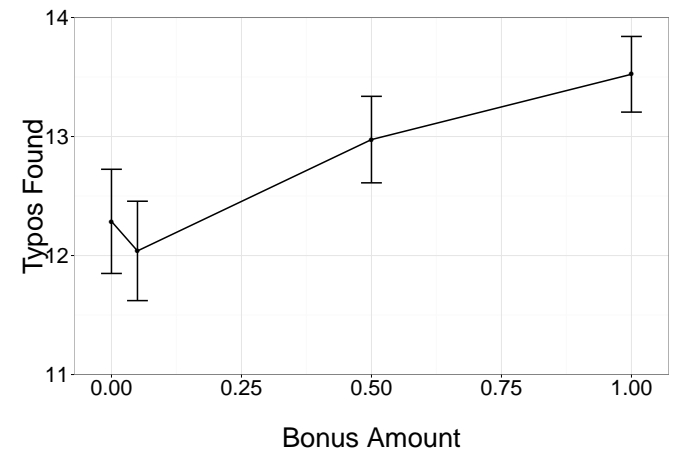

Figure 3: The effect of the bonus amount on work quality in the proofreading task. Error bars indicate the mean \pm one standard error.

This may help to explain previous negative results on PBPs in crowdsourcing markets. Shaw et al. 33 reported little or no quality improvement using PBPs compared with 
fixed payments. However, they offered a bonus payment of only $\$ 0.03,10 \%$ of their $\$ 0.30$ base. As we observe from the leftmost two points in Figure 3 PBPs do not improve quality when the bonus is very small. Yin et al. 36] tested PBPs with several bonus sizes and reported that bonus size alone did not significantly impact quality. However, their study did not include a control with fixed payments, and the bonus sizes that they tested were all significantly larger than their base payment $(\$ 0.04, \$ 0.08, \$ 0.16$, and $\$ 0.32$ on a base payment of $\$ 0.01$ ). As we observe from the rightmost two points in Figure 3 increasing the bonus size only leads to minor improvements in quality once the bonus is already sufficiently large. It is only when we view the whole picture that we can see that PBPs do help.

Taken as a whole, the results in this section show that PBPs improve quality for a wide range of possible thresholds, provided that the requester offers a bonus that is high enough to make the extra reward salient.

\section{WHY DOES PBP WORK?}

There are two primary motivations for our next experiment. First, we wanted to verify that PBPs are useful in other tasks beyond finding typos. Second, we wanted to explore potential reasons why PBPs work. In particular, as pointed out in Section 3.2, simply increasing the amount of the bonus payment led to almost as much of an improvement as using PBPs in the proofreading experiment. While it could be that workers are responding rationally to the provided incentives, it could also be the case that workers are increasing their effort due to a reciprocity effect; workers are pleasantly surprised to discover the opportunity to receive a (performance-based or unconditional) bonus after accepting the HIT, and reward the requester for this kind action by working harder. Indeed, Gilchrist et al. [10] found, in a different crowdsourcing context, that workers who accept a task and then receive an unexpected bonus do higher quality work than workers who are paid the same amount total but are told up front. This experiment is designed to test whether this "unexpected bonus effect," is the (partial) cause of the observed increases in performance using PBPs.

\subsection{Experiment Design}

In this task, workers were shown twenty pairs of images. Ten of the pairs were identical images, while the other ten pairs contained minor differences. Workers were asked to specify whether each pair was identical or not, and were not told how many pairs of images were identical in advance. Again, this task has two key properties we desire. First, we speculated that workers would be more likely to spot the differences between images if they spent more time and effort looking. Second, we can objectively measure the quality of workers' output by the number of correctly answered pairs. A similar task was used in experiments by Yin et al. [36.

To test our research questions, we wished to vary the bonus amount and bonus rules as well as the amount of the base payment. Obviously, if we launched two HITs that differed only in the base payment amount, the majority of workers would choose the HIT with the higher base payment, resulting in selection bias. To avoid this, we used the following method for randomly assigning treatments. We first posted a qualification HIT in which workers were paid a small amount $(\$ 0.05)$ if they agreed to receive notifications about our future tasks. We made it clear that they were un- der no obligation to do the future tasks. We then randomly assigned the workers who completed this recruitment task to different treatments. For each treatment, we posted a separate HIT. Workers were only qualified to see and do the HIT corresponding to their assigned treatment. Finally, we used the notifyWorkers API call to send notification emails to workers with a link to their assigned HITs (treatments). While others have suggested using qualifications to filter out workers for experiments [7] or recruiting a panel of workers in advance [28, we believe that the approach described here is novel and of independent interest.

We next describe the treatments:

- Low Base: The base payment was $\$ 0.50$. No opportunity for a bonus was given. This was our control.

- High Base: The base payment was $\$ 1.50$. No opportunity for a bonus was given.

- Unexpected Bonus: The base payment was $\$ 0.50$. After accepting the HIT, workers were told they would receive an additional bonus of $\$ 1$.

- PBP: The base payment was $\$ 0.50$. In addition to the base payment, workers could earn a bonus of $\$ 1$ if they correctly labeled $80 \%$ of the image pairs as identical or not. Workers were informed of the bonus and rules for receiving the bonus before accepting the HIT.

Note that the payment amounts in the High Base and Unexpected Bonus treatments are the same. The difference is only how and when the payments were described.

\subsection{Results}

We randomly chose 800 workers from the pool that completed the qualification HIT and randomly assigned them to the four treatments, 200 workers per treatment. After assigning qualifications corresponding to each treatment, we posted the HITs for each simultaneously and sent each worker a notification with a link to their treatment's HIT. We conducted a chi-squared test to check for significant differences in the number of participants who finished the four treatments and found none $(p=0.90)$. In the analysis we make six comparisons, described below. We did this analysis using an ANOVA with one-sided, planned comparisons 32 and report p-values that have been corrected for these multiple comparisons. The results are shown in Figure 4.

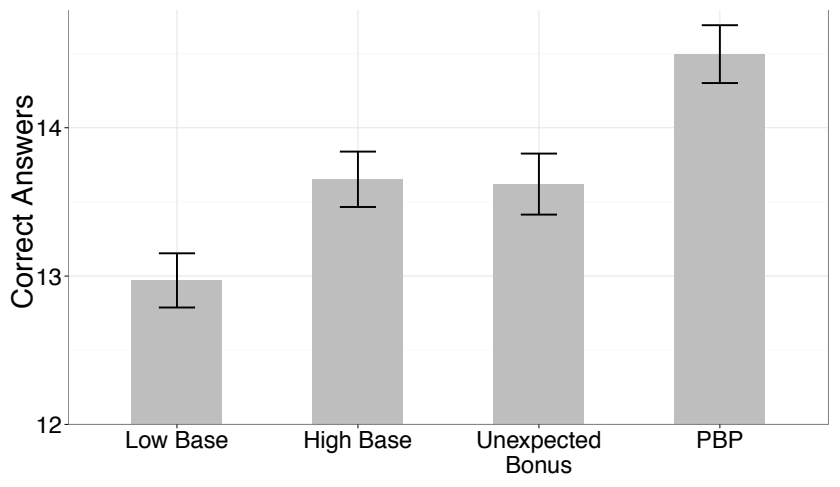

Figure 4: The effect of different payment schemes on work quality in the spot the differences task. Error bars indicate the mean \pm one standard error. 
Similar to the proofreading experiment described in Section 3 simply paying more resulted in higher quality work. The High Base treatment had a significantly higher number of correct answers than the Low Base treatment $(p=0.030)$. Similarly, the Unexpected Bonus treatment had a significantly higher number of correct answers than the Low Base treatment $(p=0.047)$. Figure 4 shows no significant difference between the High Base and the Unexpected Bonus treatments. This suggests that there was no "unexpected bonus effect" in contrast to Gilchrist et al. [10 $]^{4}$ The absence of any reciprocity effect due to the unexpected bonus suggests that workers were doing better work to increase the probability (according to their prior assumptions) that their work got accepted and thus earn the higher pay.

We also observe that workers in the PBP treatment outperformed workers in all other treatments $(p<0.005)$. This suggests that workers are rational to some degree and are willing to exert more effort to increase their chances of receiving higher payments. Note that in this experiment workers knew before they accepted the HIT that they could earn a bonus, in contrast to the experiment described in Section 3 in which workers were informed of the opportunity to earn a bonus only after they accepted the HIT. We have therefore shown that PBPs can work whether or not the opportunity for a bonus is unexpected.

\section{WHERE DOES PBP WORK?}

We have shown that PBPs incentivize higher quality crowdwork on two specific tasks, proofreading and spotting differences in images. It is natural to ask whether our results generalize, and in particular, what properties of a task open up the possibility of performance improvements with PBPs.

Camerer and Hogarth [5] note that in the context of economics lab experiments, performance-based incentives tend to improve quality for effort-responsive tasks, tasks for which it is possible to generate higher quality work by exerting additional effort (presumably without requiring too much effort). One might ask if the same is true in a crowdsourcing setting. More specifically, we investigate a hypothesis that whether, and to what extent, a task is effort-responsive is an important reason for whether or not PBPs work for this task. We find an empirical correlation between the two, which we interpret as a strong evidence in favor of this hypothesis. Since it is difficult to directly measure how much effort a worker has put into a task, we use the time a worker spent on a HIT as a proxy measure for effort, and examine the relationship between time spent and quality of work.

Figures 5(a) and 5(b) illustrate this correlation for the proofreading and spot-the-difference tasks respectively. Each shows the amount of time that a worker spent on the HIT versus the quality of his work (measured as number of typos found or number of correctly labeled image pairs as before). For the proofreading task, regressing the number of correct typos found on the amount of time a worker spent shows that every minute is correlated with finding another 0.42 typos on average $(p<0.001)$. Similarly, for the spot-the-difference task, regressing the number of pairs correctly identified on the amount of time a worker spent shows that every minute

\footnotetext{
4 Note that our experimental setting is not the same as theirs. They ran experiments on oDesk for tasks with much longer working hours ( 3 hours) while our experiment is on MTurk and our tasks only last for on average 8.8 minutes.
}

is correlated with another 0.17 correct answers $(p<0.001)$. We see that, in general, workers who spent more time on our tasks generated better quality work. We observe similar trends in all treatments, but include only workers in the control groups in the plots since they are most comparable across tasks. This is evidence that the tasks on which we observed improvements from PBPs are effort-responsive.

To further explore this hypothesis and the generalizability of our results, we examined the effects of PBPs on two additional tasks, handwriting recognition and audio transcription. For consistency with our previous experiments, we maintained a base payment of $\$ 0.50$ (adjusting the task lengths to maintain an hourly rate of roughly $\$ 6$ ) and a bonus of $\$ 1$ when applicable. Workers were randomly assigned to treatments after accepting the HIT in the same way as in the experiments described in Sections 3 and 4

\subsection{Handwriting Recognition: Design}

For the handwriting recognition task, workers were shown two images containing handwritten text and asked to transcribe the text. The images were collected from the IAM Handwriting Database 27]. One contained 89 words and the other 74. As in our other experiments, workers in the control treatment received only the base payment. We also used a PBP treatment in which workers were told that for one of the images, their transcription would be compared against a gold standard solution and they would receive the bonus if they correctly transcribed $90 \%$ of the words in the image. They were not told which image would be used to assess their accuracy. This payment rule was used to make the task appear more like a realistic MTurk task.

\subsection{Handwriting Recognition: Results}

Data was collected from 220 workers. As shown in Figure 5(c) the quality of work produced was not significantly correlated with the time a worker spent on the task. In other words, this task does not appear to be effort-responsive. Moreover, we did not find a significant difference between the accuracy of workers in the control group versus the PBP treatment via a one-sided t-test. We note that one of the two images given to workers was chosen because it was especially difficult for workers in a pilot study. Restricting our analysis to just this image did show a marginal effect, with workers in the control group averaging $92.3 \%$ accuracy and workers in the PBP treatment averaging 93.5\% $(p=0.055)$. Since PBPs only had a small, marginal effect on even the most difficult handwriting recognition image, we conclude that PBPs only have a small effect on this task in general.

One reason why PBPs did not have a strong effect on quality for this task may be that there was a ceiling effect, as discussed in the context of incentives in lab experiments by Camerer and Hogarth 5. As Figure 5(c) shows, the average accuracy over the two articles of the workers in the control group was $95.2 \%$, leaving little room for PBPs to have impact. A related explanation is that most of handwritten words in our data sets were trivial to recognize. Over $80 \%$ of words were correctly transcribed by over $90 \%$ of workers.

It is possible that PBPs would have improved performance if we had chosen a different threshold. However, since the average performance was already very high, there was little room for experimentation. If a better threshold exists, it would be difficult for a requester to identify. 


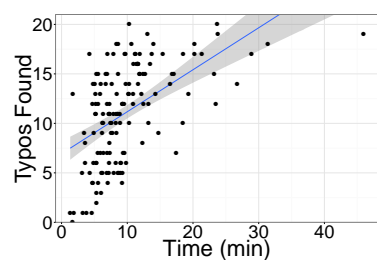

(a) Proofreading

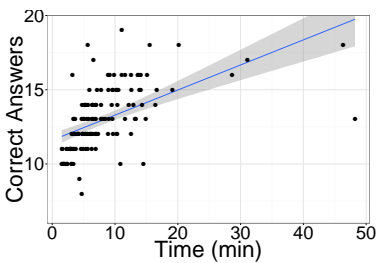

(b) Spot-the-Difference

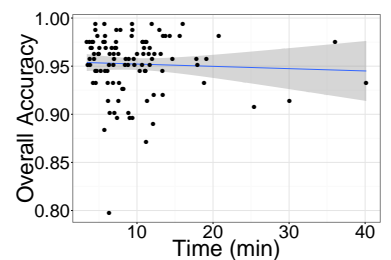

(c) Handwriting Rec.

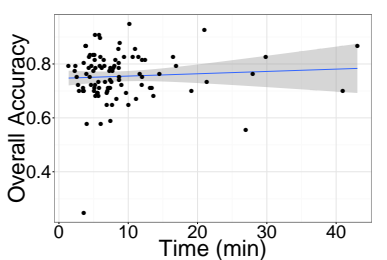

(d) Audio Transcription

Figure 5: Time vs. quality for effort responsive tasks in panels $5(\mathrm{a})$ and $5(\mathrm{~b})$, and non-effort responsive tasks in panels 5(c) and $5(\mathrm{~d})$, The blue lines indicate the regression line and the shaded areas represent the $95 \%$ confidence interval around it. Results are similar when outliers are excluded from the analysis.

\subsection{Audio Transcription: Design}

For the audio transcription task, workers were asked to transcribe 10 audio clips, each of which contained approximately 5 seconds of speech. The audio clips were collected from VoxForg $\AA^{5}$ and we intentionally chose clips from speakers with heavy accents to increase the difficulty of the task and avoid the ceiling effect. Once again workers in the control treatment received only the base payment. We additionally included three PBP treatments with different thresholds of quality required to receive the bonus. In these treatments, workers were told that their answers for 5 of the 10 clips would be compared against gold standard answers, and that they would receive the bonus if they correctly transcribed $80 \%, 85 \%$, or $90 \%$ of the words respectively. These threshold values were chosen based on quality observed in a pilot experiment on this task in order to cover the range of thresholds that we believed would be most likely to lead to quality improvements with PBPs. We note that audio transcription is one of the most common tasks on MTurk.

\subsection{Audio Transcription: Results}

We collected data from 400 workers. As Figure $5(\mathrm{~d})$ shows, quality and time are not significantly correlated. That is, audio transcription does not appear to be effort-responsive. Furthermore, we did not find a significant difference between any of the three PBP treatments and the control group. Since the average accuracy in the control group was only $75.4 \%$, this cannot be fully explained by a ceiling effect as may have been the case for handwriting recognition. One might ask if there are certain hard words for which PBPs did improve performance. We took a closer look at the data and found that this does not appear to be the case. Of the 97 words included in the 10 clips, workers' combined accuracy was better in the $85 \%$ threshold PBP treatment compared with the control on 52 words, and better in the control than the $85 \%$ threshold PBP treatment on 44 ; essentially these differences appear to be mostly due to noise. We observe a similar pattern for the other PBP treatments. Additionally, for more than $80 \%$ of the words, the percentage of workers correctly transcribing the word when PBPs were offered is close (within $\pm 5 \%$ ) to the percentage in the control group. This could suggest that workers' performance is limited by their abilities and cannot be improved through PBPs.

\subsection{A Practical Recommendation}

While the results in this section are not causal, they are in line with the hypothesis that the extent to which a task is

\footnotetext{
${ }^{5}$ http://www.voxforge.org/
}

effort-responsive is an important reason for whether or not PBPs help improve quality for this task. This suggests an approach that requesters can use when deciding whether or not to employ PBPs in their own HIT. A requester could run a pilot of their HIT with a small number of workers and a fixed (not performance-based) payment and plot the time that workers spend on the task versus the quality of their work to determine whether and to what extent the task is effort-responsive. A requester may be able to incentivize higher quality using PBPs only if the task is (sufficiently) effort-responsive. In this case, the requester must determine whether the boost in quality is worth the extra cost of PBPs.

\section{A THEORY OF WORKER INCENTIVES}

The worker model in the well-known principal-agent framework 23. assumes that a worker chooses a level of effort or quality to maximize his expected utility, which is simply the expected payment he receives minus the expected cost of doing the work at the chosen effort level. In this section, we show that incorporating the worker's subjective beliefs about how much quality is required to earn the base and bonus payments into the worker model allows us to explain the main observations from our experiments in a parsimonious way. Furthermore, it is not clear how one might capture our experimental results using the traditional worker model without incorporating the worker's subjective beliefs. Our model can be used to reason about the possible consequences of using performance-based payments.

We assume that a worker views a task and chooses to produce work at a particular quality level in order to maximize his expected utility, defined as his perceived expected payment minus the perceived cost of doing the task at a given quality level. In our model, when workers are offered performance-based payments with base payment $p$ and bonus payment $b$, the worker's expected utility is

$$
U_{\mathrm{pbp}}(q)=p \operatorname{Pr}(\text { base } \mid q)+b \operatorname{Pr}(\text { bonus } \mid q)-c(q),
$$

where $q$ is the quality level, $\operatorname{Pr}($ base $\mid q)$ and $\operatorname{Pr}($ bonus $\mid q)$ denote the worker's perceived probabilities of receiving the base and bonus payments with work of quality $q$, and $c(q)$ is the worker's perceived cost of producing quality $q$. We posit that $q$ comes from a totally ordered set, and assume that $\operatorname{Pr}($ base $\mid q)$ and $\operatorname{Pr}$ (bonus $\mid q$ ) are non-decreasing in $q$.

While we typically think of the cost of producing work as positive, capturing the effort the worker must exert to produce work of the chosen quality level, it could in some cases be negative, capturing the subjective intrinsic utility the worker receives from his enjoyment of the task or his 
satisfaction from a job well done. To allow for such effects, we make no assumptions on the monotonicity of $c(q)$.

We make a minor consistent tie-breaking assumption. If multiple quality levels maximize the expected utility for a pair of base and bonus payments, we assume the tie is broken consistently in the sense that the worker chooses the same quality level for any payments leading to this particular tie.

\subsection{Consequences of the Worker Model}

Given the worker model, we are able to provide a coherent explanation of our key observations, including some that are not explained by the standard principal-agent model. To explain the key observations from our experiments we compare Equation [1] which describes the worker's utility when there are both a base payment and a bonus payment, with the utility of a worker with subjective beliefs under the "standard" payment scheme in which workers are offered a base payment only. The standard payment scheme was used in the control group in our experiments. In this case, the worker's expected utility is simply

$$
U_{\text {std }}(q)=p \operatorname{Pr}(\text { base } \mid q)-c(q) .
$$

Let $q_{\text {std }}$ be the quality level chosen by the worker under this utility function, so $q_{\mathrm{std}} \in \arg \max _{q} U_{\mathrm{std}}(q)$, and $q_{\mathrm{pbp}}$ be the quality chosen under Equation 1 , so $q_{\mathrm{pbp}} \in \arg \max _{q} U_{\mathrm{pbp}}(q)$.

Subjective beliefs about acceptance criteria increase quality. In Section 3, we experimentally showed that the quality of work produced is higher when workers have subjective beliefs about acceptance criteria than when the base payment is explicitly guaranteed. We called this an implicit $P B P$. It is easy to explain in our model.

Consider the standard setting (no PBPs). With a guaranteed base payment, the worker's utility becomes $p-c(q)$. Let $q^{*}$ be a maximizer of this expression. It follows that $q^{*}$ is a minimizer of $c(q)$, thus $c\left(q^{*}\right) \leq c(q)$ for all $q$. For any $q<q^{*}$, we have $p \operatorname{Pr}($ base $\mid q) \leq p \operatorname{Pr}\left(\right.$ base $\left.\mid q^{*}\right)$ given the monotonicity of $\operatorname{Pr}($ base $\mid q)$. Therefore, for any $q<q^{*}$, $U_{\text {std }}(q) \leq U_{\text {std }}\left(q^{*}\right)$. Given the consistent tie-breaking assumption, the worker will not choose to generate work of quality $q$ if $q<q^{*}$. Therefore, if $q_{\text {std }}$ is the maximizer of Equation 2 then $q_{\text {std }} \geq q^{*}$. Thus $q_{\text {std }}$, the optimal quality level with no guaranteed base payment, is greater than or equal to $q^{*}$, the optimal quality level with guaranteed base payment, which we observed experimentally in Figure 1 . It is possible to strengthen this result to strict inequality $\left(q_{\text {std }}>q^{*}\right)$ with a few additional assumptions ${ }^{6}$ Similar arguments can be made for the setting with PBPs.

Higher payments increase quality. The experiments in Sections 3 and 4 demonstrated that increasing the base payment (or the unconditional bonus) can increase quality. This is also easy to explain in our model. Consider increasing the base payment in the standard setting (no PBPs). Let $q_{\delta}$ be the maximizer of $U_{\delta}(q)=(p+\delta) \operatorname{Pr}($ base $\mid q)-c(q)$, the

\footnotetext{
${ }^{6}$ In multiple places in this section, to make inequalities on quality strict, we could first assume that $q$ takes values on some interval $\left[q_{\min }, q_{\max }\right]$ such that $c\left(q_{\max }\right)>b+p$, and that $\operatorname{Pr}($ base $\mid q), \operatorname{Pr}($ bonus $\mid q)$, and $c(q)$ are differentiable on this interval. We must then also assume that the subjective probabilities $\operatorname{Pr}($ base $\mid q)$ and $\operatorname{Pr}$ (bonus $\mid q$ ) are strictly increasing in $q$. In this particular instance, under these assumptions, since $c^{\prime}\left(q^{*}\right)=0$, we have $U_{\text {std }}^{\prime}\left(q^{*}\right)>0$, which implies that $q^{*}$ cannot be the maximizer of $U_{\text {std }}^{\prime}\left(q^{*}\right)$, so $q_{\text {std }}>q^{*}$.
}

utility of the worker if the base payment were $p+\delta$ instead of $p$, where $\delta>0$. By optimality of $q_{\mathrm{std}}$, for all $q$,

$$
p \operatorname{Pr}(\text { base } \mid q)-c(q) \leq p \operatorname{Pr}\left(\text { base } \mid q_{\text {std }}\right)-c\left(q_{\text {std }}\right) .
$$

Since $\operatorname{Pr}$ (base $\mid q$ ) is non-decreasing in $q, q<q_{\text {std }}$ implies $\delta \operatorname{Pr}($ base $\mid q) \leq \delta \operatorname{Pr}$ (base $\left.\mid q_{\text {std }}\right)$. Combining the last two inequalities, $U_{\delta}(q) \leq U_{\delta}\left(q_{\mathrm{std}}\right)$ for all $q<q_{\mathrm{std}}$. Therefore, given the consistent tie-breaking assumption, $q_{\delta}$, the optimal quality level under higher pay, is greater than or equal to $q_{\text {std }}$, the optimal quality level under the standard setting. Again, strict inequality is achievable with the additional assumptions from Footnote 6 Similar arguments can be made for increasing the base payment with PBPs, and for increasing the bonus payment.

These conclusions hold only when uncertainty about receiving the payment is included. They would not hold in the standard principal-agent model, where increasing a guaranteed payment does not increase quality.

Performance-based payments (significantly) increase quality. A key result from our experiments in Sections 35 is that PBPs can, in fact, increase quality in a significant way. This can be explained from our model too.

We have that the quality improves $\left(q_{\mathrm{pbp}} \geq q_{\mathrm{std}}\right.$, or $q_{\mathrm{pbp}}>$ $q_{\text {std }}$ with additional assumptions) as a special case of the argument above. However, this statement is relatively weak, as it does not say anything about the magnitude of the improvement, i.e., the difference $q_{\mathrm{pbp}}-q_{\mathrm{std}}$. We would like this difference to be large; a requester might not want to take on the extra costs of PBPs if the increase in quality is small. In order to gain some intuition for when it is or is not possible to obtain a sufficiently large improvement in quality, we consider several special cases as examples.

As a first example, consider the case in which the worker does not have fine-grained control over the precise quality of his work, but can only choose between two options: highquality, denoted $q_{\text {high }}$, or low quality, $q_{\text {low }}$. Then PBPs work if and only if the worker's optimal quality level is $q_{1 \text { ow }}$ without PBPs, and $q_{\text {high }}$ with PBPs. In formulas, $U_{\text {std }}\left(q_{\text {high }}\right)<$ $U_{\text {std }}\left(q_{\text {low }}\right)$ and $U_{\text {pbp }}\left(q_{\text {high }}\right)>U_{\text {pbp }}\left(q_{\text {low }}\right)$, or

$$
\begin{gathered}
p\left(\operatorname{Pr}\left(\text { base } \mid q_{\text {high }}\right)-\operatorname{Pr}\left(\text { base } \mid q_{\text {low }}\right)\right)<c\left(q_{\text {high }}\right)-c\left(q_{\text {low }}\right) \\
<p\left(\operatorname{Pr}\left(\text { base } \mid q_{\text {high }}\right)-\operatorname{Pr}\left(\text { base } \mid q_{\text {low }}\right)\right)+ \\
b\left(\operatorname{Pr}\left(\text { bonus } \mid q_{\text {high }}\right)-\operatorname{Pr}\left(\text { bonus } \mid q_{\text {low }}\right)\right) .
\end{gathered}
$$

In words, the extra cost to produce high-quality work must be less than the extra benefit the worker would receive in terms of expected payments if the bonus is included, and bigger than the extra benefit he would receive with standard payments. Examining this expression gives us intuition about when we might expect PBPs to help. First, $c\left(q_{\mathrm{high}}\right)-c\left(q_{\text {low }}\right)$ cannot be too large. It must be possible for the worker to substantially increase his quality with additional effort (and at a reasonable cost) - essentially, the task must be effort-responsive, as conjectured in Section 6 Second, $c\left(q_{\text {high }}\right)-c\left(q_{\text {low }}\right)$ cannot be too small (in particular, compared with $\operatorname{Pr}\left(\right.$ base $\left.\mid q_{\text {high }}\right)-\operatorname{Pr}\left(\right.$ base $\left.\mid q_{\text {low }}\right)$ and $\left.p\right)$ or PBPs are unnecessary to achieve high quality. This is a partial explanation for why we did not see improvements from PBPs in the handwriting recognition task when the cost of producing high quality was already small. Third, the bonus $b$ must be set large enough. This could partially explain our observation that PBPs did not help in the proofreading task with a very small bonus. Finally, the difference 
$\operatorname{Pr}$ (bonus $\left.\mid q_{\text {high }}\right)-\operatorname{Pr}$ (bonus $\left.\mid q_{\text {low }}\right)$ must be high enough. This could explain why we observed that PBPs did not help when the threshold for receiving a bonus is set too low.

As a second tractable example, suppose that the worker has fine-grained control over quality, but has no uncertainty over whether the bonus will be obtained, i.e.,

$$
\operatorname{Pr}(\text { bonus } \mid q)= \begin{cases}1 & \text { if } q \geq \bar{q} \\ 0 & \text { otherwise }\end{cases}
$$

for some threshold value $\bar{q}$. It is easy to show that either $q_{\mathrm{pbp}}=q_{\mathrm{std}}$ (if the worker prefers to do less work and pass up the bonus) or $q_{\mathrm{pbp}} \geq \bar{q}$ (if the worker prefers to do more work to receive the bonus). PBPs are useful if and only if $\bar{q}$ is sufficiently higher than $q_{\text {std }}$ (according to the needs of the requester), and for some $q \geq \bar{q}, U_{\mathrm{pbp}}(q)>U_{\mathrm{pbp}}\left(q_{\mathrm{std}}\right)$, or equivalently

$$
c(q)-c\left(q_{\mathrm{std}}\right)<p\left(\operatorname{Pr}(\text { base } \mid q)-\operatorname{Pr}\left(\text { base } \mid q_{\text {std }}\right)\right)+b .
$$

Again we see that for PBPs to help it must be possible for the worker to increase his quality with additional effort at a reasonable cost, and the bonus must be set sufficiently large.

Equation 3 provides a concrete and simple way to think about whether it would help to increase the bonus threshold $\bar{q}$. For fixed payments $p$ and $b$, it is optimal to choose as $\bar{q}$ the largest $q$ which satisfies Equation 3 However, $\bar{q}$ is a perceived threshold and cannot always be controlled directly. Instead, it may help to alter workers' perception, perhaps without even changing the objective bonus rule. For example, in the proofreading experiment from Section 4 the "5 typos" bonus rule is roughly equivalent to the " $25 \%$ " bonus rule in terms of when bonus payments are awarded, yet workers react differently. One possible explanation for this general phenomenon is a difference in the perceived value $\bar{q}$.

\subsection{Comparison with Principal-Agent Model}

In the standard principal-agent model, a worker maximizes his payment minus the intrinsic cost of his effort. We deviate from the standard model in that we include the worker's subjective beliefs about how high quality his work must be to be paid. In particular, while the objective probability of receiving the base payment is often 1 , the subjective probability may be much smaller, depending on the quality level. This feature allows us to capture some effects that are not captured by the standard model, e.g., that increasing the base payment may increase the quality of work that the worker chooses to produce, while removing uncertainty about the base payment may decrease quality.

Our model may be useful as a more realistic foundation for theoretical work. As an example, consider Ho et al. [16], a recent theoretical paper on the optimization of PBPs. While that paper posits the standard principal-agent model, all results carry over to our model. (A proof of this fact is omitted due to space constraints.)

\section{CONCLUSIONS AND DISCUSSION}

We describe the results of a series of experiments studying the effect of performance-based payments on the quality of crowdwork on Amazon Mechanical Turk. Our goal is to identify properties of the payment, payment structure, and task that allow for quality improvements using PBPs.

We find that PBPs can improve the quality of submitted work for some tasks, but are not likely to for others.
We identify the extent to which a task is effort-responsive as a potential important reason for whether or not PBPs work for this task. This leads to an actionable insight for requesters. When considering whether or not to use PBPs, one could first run a pilot experiment to determine whether a task is effort-responsive by examining the correlation between time spent and quality. If additional time spent leads to a sufficiently high boost in quality, we would expect PBPs to improve performance.

We find strong evidence for what we call the implicit $P B P$ effect: workers may have their own subjective beliefs about the quality of work they must submit to have their work accepted, which makes them view fixed payments as implicitly performance-based. Workers may also have subjective beliefs about the likelihood of receiving the bonus when payments are explicitly performance-based. This should be taken into account when designing a payment scheme. For example, we find that in some cases a requester can incentivize higher quality work by defining the bonus threshold relative to other workers or with respect to gold standard data that the workers do not have access to.

We show that in order for PBPs to improve quality, the bonus payment offered must be sufficiently large, but that there are diminishing returns for further increasing this payment. This partially explains existing negative results on the effectiveness of PBPs in crowdsourcing markets. We provide evidence that when PBPs improve quality, they do so for a wide range of quality thresholds.

Finally, we suggest a theoretical model of workers' behavior that captures all of the above effects and explains several outcomes we observed in our experiments. We believe this model may be useful in further work on crowdsourcing markets, both as a concrete way to think about the consequences of using this or that payment scheme in practice, and as a more realistic foundation for theoretical work compared to the standard principal-agent model.

\section{Acknowledgements}

This research was partially supported by the National Science Foundation under grant IIS-1054911.

\section{References}

[1] O. Alonso. Implementing crowdsourcing-based relevance experimentation: An industrial perspective. Information Retrieval, 16(2):101-120, 2013.

[2] R. M. Araujo. 99designs: An analysis of creative competition in crowdsourced design. In $H C O M P$, 2013.

[3] S. E. Bonner, R. Hastie, S. G. B., and S. M. Young. A review of the effects of financial incentives on performance in laboratory tasks: Implications for management accounting. Journal of Management Accounting Research, 12(1):19-64, 2000.

[4] M. Buhrmester, T. Kwang, and S. D. Gosling. Amazon's Mechanical Turk: A new source of inexpensive, yet high-quality, data? Perspectives on Psychological Science, 2011.

[5] C. F. Camerer and R. Hogarth. The effects of financial incentives in economics experiments: A review and capital-labor-production framework. Journal of Risk and Uncertainty, 19(1):7-42, 1999. 
[6] J. Cameron and W. D. Pierce. Reinforcement, reward, and intrinsic motivation: A meta-analysis. Review of Educational Research, 64(3):363-423, 1994.

[7] J. Chandler, P. Mueller, and G. Paolacci. Nonnaïveté among Amazon Mechanical Turk workers: Consequences and solutions for behavioral researchers. Behavior Research Methods, 46(1):112-130, 2014.

[8] R. Eisenberger and J. Cameron. Detrimental effects of reward: Reality or myth? American psychologist, 51 (11):1153, 1996.

[9] B. S. Frey and F. Oberholzer-Gee. The cost of price incentives: An empirical analysis of motivation crowding-out. The American Economic Review, pages 746-755, 1997.

[10] D. Gilchrist, M. Luca, and D. Malhotra. When $3+1>$ 4: Gift structure and reciprocity in the field. Technical report, 2014. Working Paper.

[11] U. Gneezy and A. Rustichini. Pay enough or don't pay at all. Quarterly Journal of Economics, August, pages 791-810, 2000.

[12] C. G. Harris. You're hired! An examination of crowdsourcing incentive models in human resource tasks. In WSDM 2011 Workshop on Crwdsourcing for Search and Data Mining, 2011.

[13] R. Hertwig and A. Ortmann. Experimental practices in economics: A methodological challenge for psychologists? Behavioral and Brain Sciences, 24(3): 383-403, 2001.

[14] C.-J. Ho and J. W. Vaughan. Online task assignment in crowdsourcing markets. In $A A A I, 2012$.

[15] C.-J. Ho, S. Jabbari, and J. W. Vaughan. Adaptive task assignment for crowdsourced classification. In ICML, 2013.

[16] C.-J. Ho, A. Slivkins, and J. W. Vaughan. Adaptive contract design for crowdsourcing markets: Bandit algorithms for repeated principal-agent problems. In ACM EC, 2014.

[17] J. J. Horton and L. B. Chilton. The labor economics of paid crowdsourcing. In ACM EC, 2010.

[18] J. J. Horton, D. Rand, and R. Zeckhauser. The online laboratory: Conducting experiments in a real labor market. Experimental Economics, 14(3):399-425, 2011.

[19] P. G. Ipeirotis, F. Provost, and J. Wang. Quality management on Amazon Mechanical Turk. In HCOMP, 2010.

[20] G. D. Jenkins Jr., N. Gupta, A. Gupta, and J. D. Shaw. Are financial incentives related to performance? A meta-analytic review of empirical research. Journal of Applied Psychology, 83(5):777-787, 1998.
[21] D. Karger, S. Oh, and D. Shah. Iterative learning for reliable crowdsourcing systems. In NIPS, 2011.

[22] A. Kittur, E. Chi, and B. Suh. Crowdsourcing user studies with Mechanical Turk. In CHI, 2008.

[23] J.-J. Laffont and D. Martimort. The Theory of Incentives: The Principal-Agent Model. Princeton University Press, 2002.

[24] E. P. Lazear. Performance pay and productivity. American Economic Review, pages 1346-1361, 2000.

[25] L. Litman, J. Robinson, and C. Rosenzweig. The relationship between motivation, monetary compensation, and data quality among US- and India-based workers on Mechanical Turk. Behavioral Research Methods, 2014. To appear.

[26] Q. Liu, J. Peng, and A. Ihler. Variational inference for crowdsourcing. In NIPS, 2012.

[27] U.-V. Marti and H. Bunke. The iam-database: an english sentence database for offline handwriting recognition. International Journal on Document Analysis and Recognition, 5(1):39-46, 2002.

[28] W. Mason and S. Suri. Conducting behavioral research on Amazon's Mechanical Turk. Behavior Research Methods, 44(1):1-23, 2012.

[29] W. Mason and D. J. Watts. Financial incentives and the "performance of crowds". In HCOMP, 2009.

[30] J. Rogstadius, V. Kostakos, A. Kittur, B. Smus, J. Laredo, and M. Vukovic. An assessment of intrinsic and extrinsic motivation on task performance in crowdsourcing markets. In ICWSM, 2011.

[31] D. Schall. Service-Oriented Crowdsourcing Architecture, Protocols and Algorithms. Springer Briefs in Computer Science. Springer, 2012.

[32] H. J. Seltman. Experimental design and analysis. http://www. stat.cmu.edu/\{ $\}$ hseltman/309/Book 2014.

[33] A. D. Shaw, J. J. Horton, and D. L. Chen. Designing incentives for inexpert human raters. In $C S C W, 2011$.

[34] V. Sheng, F. Provost, and P. Ipeirotis. Get another label? Improving data quality using multiple, noisy labelers. In $K D D, 2008$.

[35] C. Wah, S. Branson, P. Welinder, P. Perona, and S. Belongie. The Caltech-UCSD Birds-200-2011 Dataset. Technical Report CNS-TR-2011-001, California Institute of Technology, 2011.

[36] M. Yin, Y. Chen, and Y.-A. Sun. The effects of performance-contingent financial incentives in online labor markets. In $A A A I, 2013$.

[37] M. Yin, Y. Chen, and Y.-A. Sun. Monetary interventions in crowdsourcing task switching. In HCOMP, 2014. 